\title{
Percolation of clusters with a residence time in the bond definition: Integral equation theory
}

\author{
Guillermo J. Zarragoicoechea* \\ Instituto de Física de Líquidos y Sistemas Biológicos (IFLYSIB), c.c. 565 (1900) La Plata, Argentina \\ Luis A. Pugnaloni ${ }^{\dagger}$ \\ Procter Department of Food Science, University of Leeds, LS2 9JT, United Kingdom \\ Fred Lado \\ Department of Physics, North Carolina State University, Raleigh, North Carolina 27695-8202, USA \\ Enrique Lomba \\ Instituto de Química Física Rocasolano (CSIC), Serrano 119, E-28006 Madrid, Spain \\ Fernando Vericat ${ }^{\ddagger}$ \\ Grupo de Aplicaciones Matemáticas y Estadísticas de la Facultad de Ingeniería (GAMEFI), UNLP, La Plata, Argentina
}

(Received 22 September 2004; published 18 March 2005)

\begin{abstract}
We consider the clustering and percolation of continuum systems whose particles interact via the LennardJones pair potential. A cluster definition is used according to which two particles are considered directly connected (bonded) at time $t$ if they remain within a distance $d$, the connectivity distance, during at least a time of duration $\tau$, the residence time. An integral equation for the corresponding pair connectedness function, recently proposed by two of the authors [Phys. Rev. E 61, R6067 (2000)], is solved using the orthogonal polynomial approach developed by another of the authors [Phys. Rev. E 55, 426 (1997)]. We compare our results with those obtained by molecular dynamics simulations.
\end{abstract}

DOI: 10.1103/PhysRevE.71.031202

PACS number(s): 61.20.Gy, 64.60.Ak

\section{INTRODUCTION}

The ideas of clustering and percolation have been exploited to explain numerous macroscopic properties in manybody systems and a wide class of problems has been studied using this approach. We can mention, among many others, the insulator-conductor transition [1], the glass transitions $[2,3]$ and the sol-gel transition [4] observed in several materials, the behavior of supercooled water $[5,6]$, aggregation and agglutination phenomena in cells and biological macromolecules and organelles [7-9], the flow of fluids in porous media [10], earthquakes and fractures in the terrestrial crust $[11,12]$, and the large-scale structure of the Universe [13-15]. The concept of connectivity between the particles of the system plays a central role in this type of description. Most of the efforts made in these areas are based on a lattice representation of the systems of interest. The relative simplicity of lattice models allows for a wide variety of treatments, which extend from almost heuristic [16] to quite rigorous [17].

Despite the ubiquity of the lattice representation and the contribution it has made to our understanding of many-

\footnotetext{
*Also at Comisión de Investigaciones Científicas y Tecnológicas de la Prov. de Buenos Aires (CICPBA), Argentina.

†Present address: Instituto de Física de Líquidos y Sistemas Biológicos (IFLYSIB), La Plata, Argentina.

*Also at Instituto de Física de Líquidos y Sistemas Biológicos (IFLYSIB), La Plata, Argentina.

Email address: vericat@iflysib.unlp.edu.ar
}

particle systems, sometimes a continuous description -where particles can occupy any point in a continuum phase space - is needed to reach a more realistic picture of the phenomena under consideration. Within this context, the concept of connectivity has been generalized and adapted to describe clustering and percolation in continuum systems. The main ideas have been established in the pioneering works of Hill [18] and Coniglio et al. [19]. Hill considers a partition of the whole system into subsystems of particles (the clusters) that satisfy some linking properties. The concept of cluster is thus directly related to the idea of bonded pairs. A bonded pair is a set of two particles that are linked by some direct mechanism. A cluster is then defined as a set of particles such that any pair of particles in the set is connected through a path of bonded pairs. We call these clusters "chemical clusters" to distinguish them from the non-pairbonded clusters we have introduced in a previous work [20] - note, however, that this does not mean that clusters are necessarily formed through chemical bonding. A system is said to be in a percolated configuration if it contains a cluster that spans the system.

From Hill's theory, we see that a connectivity criterion is needed in order to decide whether two particles are bonded or not. This connectivity criterion has to be defined in accord with the phenomenon under study [21-23]. In the search for stable atomic clusters, which mark the onset of a phase transition in a monatomic gas, Hill proposed a simple energetic criterion: two particles are bonded if their relative kinetic energy is less than the negative of their relative potential energy [18]. However, this criterion is difficult to implement from a theoretical point of view (see Ref. [24] for a first 
attempt to tackle this problem) and simpler criteria have been preferred. Thus, most of the theoretical studies on connectivity and percolation in continuum systems (see for example Refs. [25-27]) were focused on the rather simple geometrical connectivity criterion of Stillinger [28]. Stillinger's criterion states that two particles are bonded if they are separated by a distance shorter than a given connectivity distance $d$. In this case, $d$ is an ad hoc parameter which must be chosen on physical grounds.

The use of a geometrical criterion to decide whether two particles are bonded might be meaningful in some applications where the fact that two particles are close together at a given time is sufficient to infer the existence of a particleparticle bond. However, in any real experiment, a cluster and the particle-particle bonds that build it up need to last for some minimum period of time in order to be detected. Examples where the finite value of the bond lifetime is of crucial importance to the understanding of the clustering and percolation phenomena include the formation of hydrogen bonds in liquid and glassy water $[6,29]$ and the viscoelastic sol-gel transition [30].

In a previous work [31] (hereafter denoted as I), two of us presented a generalized connectedness integral equation theory for continuum systems - and molecular dynamics (MD) simulations for the Lennard-Jones system [20] where the finite lifetime of the particle-particle bonds was explicitly considered. In this generalization two particles are considered bonded at time $t$ if they remain within a distance $d$ (the connectivity distance) at least during a period of time of length $\tau$ (the residence time). This connectivity criterion allows us to detect bonds with different lifetimes by simply setting $\tau$ to any nonzero value. The clusters so obtained are called "chemical clusters" to distinguish them from the "physical clusters" [20] which have no need of bonded pairs to exist.

The solution of the integral equation derived from this theory posed an important challenge since it involves convolutions not only on the positions but also on the momenta of the particles. The form of the integral equation turned out to be mathematically equivalent to that used to study the structure and thermodynamics of a model for nonpolar, polarizable molecules $[32,33]$. In this paper, we adapt the technique of expansion in orthogonal polynomials, developed by one of us in Ref. [33] (hereafter denoted as II) in the study of polarizable molecules, to solve the connectedness integral equation at nonzero bond lifetime for the Lennard-Jones system. We compare the chemical-cluster pair correlation function $g_{\text {chem }}\left(\mathbf{r}_{1}, \mathbf{r}_{2}\right)$ and the percolation line with MD simulations for the same system. The cluster pair correlation function is proportional to the joint probability density of finding two particles (labeled 1 and 2) within the same cluster and at positions $\mathbf{r}_{1}$ and $\mathbf{r}_{2}$, respectively. The percolation line in the temperature-density plane separates the phase space into a nonpercolated region (at low densities) and a percolated region (at high densities).

The rest of the paper is organized as follows. In Sec. II we summarize the generalized connectedness integral equation theory to treat nonzero bond lifetimes. In Sec. III we present the expansion in orthogonal polynomials used to solve the integral equation in the Percus-Yevick approximation. Sec- tion IV is devoted to the numerical results and a comparison with our MD simulations. We finish with conclusions and a brief description of the implications of this generalized connectivity criterion on the study of gelation in weakly attractive colloids.

\section{CHEMICAL CLUSTERS}

We summarize here the basic theory that we have developed in I to describe the clustering and percolation for chemical clusters with nonzero bond lifetime.

For a system of $N$ classical particles that interact through a pair potential $v\left(\mathbf{r}_{i}, \mathbf{r}_{j}\right)$, we define a density correlation function $\rho\left(\mathbf{r}_{1}, \mathbf{r}_{2}, \mathbf{p}_{1}, \mathbf{p}_{2}\right)$ that is $N(N-1)$ times the probability density of finding two particles at the phase space configurations $\left(\mathbf{r}_{1}, \mathbf{p}_{1}\right)$ and $\left(\mathbf{r}_{2}, \mathbf{p}_{2}\right)$, respectively:

$$
\begin{aligned}
\rho\left(\mathbf{r}_{1}, \mathbf{r}_{2}, \mathbf{p}_{1}, \mathbf{p}_{2}\right)= & \frac{N(N-1)}{h^{3 N} N ! Q(N, V, T)} \\
& \times \int \prod_{i=1}^{N} \exp \left[-\beta \frac{\mathbf{p}_{i}^{2}}{2 m}\right] \prod_{i=1}^{N} \prod_{j>i}^{N} \\
& \times \exp \left[-\beta v\left(\mathbf{r}_{i}, \mathbf{r}_{j}\right)\right] d \mathbf{r}^{N-2} d \mathbf{p}^{N-2} .
\end{aligned}
$$

Here $h$ is Planck's constant and $Q(N, V, T)$ the canonical partition function of the system. Then, in the same spirit of Hill and Coniglio et al. $[18,19]$, we separate $\exp \left[-\beta v\left(\mathbf{r}_{i}, \mathbf{r}_{j}\right)\right]$ into connecting $(\dagger)$ and blocking $(*)$ parts,

$$
\exp \left[-\beta v\left(\mathbf{r}_{i}, \mathbf{r}_{j}\right)\right]=f^{\dagger}\left(\mathbf{r}_{i}, \mathbf{r}_{j}, \mathbf{p}_{i}, \mathbf{p}_{j}\right)+f^{*}\left(\mathbf{r}_{i}, \mathbf{r}_{j}, \mathbf{p}_{i}, \mathbf{p}_{j}\right)+1 .
$$

Here $f^{\dagger}\left(\mathbf{r}_{i}, \mathbf{r}_{j}, \mathbf{p}_{i}, \mathbf{p}_{j}\right)$ represents the basic probability density that two particles in configuration $\left(\mathbf{r}_{i}, \mathbf{r}_{j}, \mathbf{p}_{i}, \mathbf{p}_{j}\right)$ are bonded. We will sometimes use the shorthand notation $f^{\gamma}\left(\mathbf{r}_{i}, \mathbf{r}_{j}, \mathbf{p}_{i}, \mathbf{p}_{j}\right) \equiv f_{i, j}^{\gamma}$, where $\gamma$ can be either $\dagger$ or $*$. Substitution of Eq. (2) in Eq. (1) yields

$$
\begin{aligned}
\rho\left(\mathbf{r}_{1}, \mathbf{r}_{2}, \mathbf{p}_{1}, \mathbf{p}_{2}\right)= & \frac{N(N-1)}{h^{3 N} N ! Q(N, V, T)} \exp \left[-\beta v\left(\mathbf{r}_{1}, \mathbf{r}_{2}\right)\right] \\
& \times \int \prod_{i=1}^{N} \exp \left[-\beta \frac{\mathbf{p}_{i}^{2}}{2 m}\right] \sum\left\{\prod f_{i, j}^{\dagger} f_{k, l}^{*}\right\} \\
& \times d r^{N-2} d p^{N-2},
\end{aligned}
$$

where the sum is carried out over all possible arrangements of products of functions $f_{i, j}^{\dagger}$ and $f_{k, l}^{*}$.

We note that the functions $f_{i, j}^{\dagger}$ and $f_{i, j}^{*}$ can depend on the momenta as well as on the positions of the two particles, but the sum of $f_{i, j}^{\dagger}$ and $f_{i, j}^{*}$ must be momentum independent in order to conform to Eq. (2). Except for this last condition, the functions $f_{i, j}^{\dagger}$ and $f_{i, j}^{*}$ are otherwise arbitrary for thermodynamic purposes. Of course, we choose them in such a way that the desired definition of bonded particles for chemical clusters is achieved, i.e.,

$$
f^{\dagger}\left(\mathbf{r}_{i}, \mathbf{r}_{j}, \mathbf{p}_{i}, \mathbf{p}_{j}\right)=\left\{\begin{array}{l}
\exp \left[-\beta v\left(\mathbf{r}_{i}, \mathbf{r}_{j}\right)\right], \quad\left|\mathbf{r}_{i, j}(t)\right|<d \quad \forall t \leqslant \tau, \\
0, \quad \text { otherwise, }
\end{array}\right.
$$




$$
f^{*}\left(\mathbf{r}_{i}, \mathbf{r}_{j}, \mathbf{p}_{i}, \mathbf{p}_{j}\right)=\left\{\begin{array}{l}
-1, \quad\left|\mathbf{r}_{i, j}(t)\right|<d \quad \forall t \leqslant \tau, \\
\exp \left[-\beta v\left(\mathbf{r}_{i}, \mathbf{r}_{j}\right)\right]-1, \quad \text { otherwise, }
\end{array}\right.
$$

where $\mathbf{r}_{i, j}(t)$ is the relative position of the particles $i$ and $j$ at time $t$ within the time interval $[0, \tau]$. We assume that $\left(\mathbf{r}_{i}, \mathbf{r}_{j}, \mathbf{p}_{i}, \mathbf{p}_{j}\right)$ is the configuration of the set at $t=0$. We see that in fact Eq. (2) is satisfied by Eqs. (4) and (5). Equation (4) states that two particles $i$ and $j$ are bonded if they remain separated by a distance shorter than $d$ for at least a time interval of length $\tau$. This coincides with our definition of chemical clusters. Time is explicitly introduced here by taking the set $\left\{\mathbf{r}^{N}, \mathbf{p}^{N}\right\}$ as initial conditions for $t=0$ and solving the equations of motion of the $N$ particles under their mutual interaction.

In order to calculate $\mathbf{r}_{i, j}(t)$ exactly for any $t$ we must solve a many-body problem. An approximation to this can be obtained by reducing the calculation to a two-body problem. This is done by using the potential of mean force $v^{\text {eff }}\left(\mathbf{r}_{i}, \mathbf{r}_{j}\right)=-\ln \left[g\left(\mathbf{r}_{i}, \mathbf{r}_{j}\right)\right] / \beta[34]$, where $g\left(\mathbf{r}_{i}, \mathbf{r}_{j}\right)$ is the system pair distribution function (PDF). In this way, $\mathbf{r}_{i, j}(t)$ is obtained in terms of just the initial values $\mathbf{r}_{i}, \mathbf{r}_{j}, \mathbf{p}_{i}$, and $\mathbf{p}_{j}$.

Each term in the integrand of Eq. (3) can be represented as a diagram consisting of two white $e_{1}$ and $e_{2}$ points, $N-2$ black $e_{i}$ points and some $f_{i, j}^{\dagger}$ and $f_{i, j}^{*}$ connections except between the white points. Here we take $e_{i} \equiv \exp \left[-\beta\left(\mathbf{p}_{i}^{2} / 2 m\right)\right]$. White points are not integrated over whereas black points are integrated over both their positions and momenta. All the machinery normally used to handle standard diagrams in classical liquid theory [32] can now be extended to treat these new type of diagrams. By following Coniglio's recipe to separate connecting and blocking parts in the PDF, $g\left(\mathbf{r}_{1}, \mathbf{r}_{2}\right)=g^{\dagger}\left(\mathbf{r}_{1}, \mathbf{r}_{2}, \mathbf{p}_{1}, \mathbf{p}_{2}\right)+g^{*}\left(\mathbf{r}_{1}, \mathbf{r}_{2}, \mathbf{p}_{1}, \mathbf{p}_{2}\right)$, we obtain an Ornstein-Zernike-like integral equation for $g^{\dagger}\left(\mathbf{r}_{1}, \mathbf{r}_{2}, \mathbf{p}_{1}, \mathbf{p}_{2}\right)$,

$$
\begin{aligned}
g^{\dagger}\left(\mathbf{r}_{1}, \mathbf{r}_{2}, \mathbf{p}_{1}, \mathbf{p}_{2}\right)= & c^{\dagger}\left(\mathbf{r}_{1}, \mathbf{r}_{2}, \mathbf{p}_{1}, \mathbf{p}_{2}\right) \\
& +\int \rho\left(\mathbf{r}_{3}, \mathbf{p}_{3}\right) c^{\dagger}\left(\mathbf{r}_{1}, \mathbf{r}_{3}, \mathbf{p}_{1}, \mathbf{p}_{3}\right) g^{\dagger}\left(\mathbf{r}_{3}, \mathbf{r}_{2}, \mathbf{p}_{3}, \mathbf{p}_{2}\right) \\
& \times d \mathbf{r}_{3} d \mathbf{p}_{3} .
\end{aligned}
$$

Here $\rho\left(\mathbf{r}_{1}, \mathbf{p}_{1}\right) \rho\left(\mathbf{r}_{2}, \mathbf{p}_{2}\right) g^{\dagger}\left(\mathbf{r}_{1}, \mathbf{r}_{2}, \mathbf{p}_{1}, \mathbf{p}_{2}\right)$ is $N(N-1)$ times the joint probability density of finding two particles at positions $\mathbf{r}_{1}$ and $\mathbf{r}_{2}$ with momenta $\mathbf{p}_{1}$ and $\mathbf{p}_{2}$, respectively, and belonging to the same cluster, where the bonding criterion is given by Eqs. (4) and (5), while

$$
\rho\left(\mathbf{r}_{1}, \mathbf{p}_{1}\right)=\frac{1}{N-1} \int \rho\left(\mathbf{r}_{1}, \mathbf{r}_{2}, \mathbf{p}_{1}, \mathbf{p}_{2}\right) d \mathbf{r}_{2} d \mathbf{p}_{2} .
$$

The function $c^{\dagger}\left(\mathbf{r}_{1}, \mathbf{r}_{2}, \mathbf{p}_{1}, \mathbf{p}_{2}\right)$ denotes the sum of all the nonnodal diagrams in the diagrammatic expansion of $g^{\dagger}\left(\mathbf{r}_{1}, \mathbf{r}_{2}, \mathbf{p}_{1}, \mathbf{p}_{2}\right)$. We recall here that a nodal diagram contains at least one black point through which all paths between the two white points pass. For a homogeneous system, we have

$$
\begin{aligned}
g^{\dagger}\left(\mathbf{r}_{12}, \mathbf{p}_{1}, \mathbf{p}_{2}\right)= & c^{\dagger}\left(\mathbf{r}_{12}, \mathbf{p}_{1}, \mathbf{p}_{2}\right)+\frac{\rho}{\left(2 \pi m k_{B} T\right)^{3 / 2}} \\
& \times \int \exp \left[-\beta \frac{p_{3}^{2}}{2 m}\right] c^{\dagger}\left(\mathbf{r}_{13}, \mathbf{p}_{1}, \mathbf{p}_{3}\right) g^{\dagger}\left(\mathbf{r}_{32}, \mathbf{p}_{3}, \mathbf{p}_{2}\right) \\
& \times d \mathbf{r}_{3} d \mathbf{p}_{3} .
\end{aligned}
$$

To obtain a closed integral equation with Eq. (6) or Eq. (8), we need a closure relation between $g^{\dagger}\left(\mathbf{r}_{1}, \mathbf{r}_{2}, \mathbf{p}_{1}, \mathbf{p}_{2}\right)$ and $c^{\dagger}\left(\mathbf{r}_{1}, \mathbf{r}_{2}, \mathbf{p}_{1}, \mathbf{p}_{2}\right)$. Here we will use the Percus-Yevick approximation $g\left(\mathbf{r}_{1}, \mathbf{r}_{2}\right) \exp \left[\beta v\left(\mathbf{r}_{1}, \mathbf{r}_{2}\right)\right]=1+N\left(\mathbf{r}_{1}, \mathbf{r}_{2}\right)$, where the function $N\left(\mathbf{r}_{1}, \mathbf{r}_{2}\right)$ is the sum of the nodal diagrams in the expansion of $g\left(\mathbf{r}_{1}, \mathbf{r}_{2}\right)$. Separation into connecting and blocking parts, $g\left(\mathbf{r}_{1}, \mathbf{r}_{2}\right)=g^{\dagger}\left(\mathbf{r}_{1}, \mathbf{r}_{2}, \mathbf{p}_{1}, \mathbf{p}_{2}\right)+g^{*}\left(\mathbf{r}_{1}, \mathbf{r}_{2}, \mathbf{p}_{1}, \mathbf{p}_{2}\right)$ and $N\left(\mathbf{r}_{1}, \mathbf{r}_{2}\right)=N^{\dagger}\left(\mathbf{r}_{1}, \mathbf{r}_{2}, \mathbf{p}_{1}, \mathbf{p}_{2}\right)+N^{*}\left(\mathbf{r}_{1}, \mathbf{r}_{2}, \mathbf{p}_{1}, \mathbf{p}_{2}\right)$, yields

$$
\begin{aligned}
g^{\dagger}\left(\mathbf{r}_{1}, \mathbf{r}_{2}, \mathbf{p}_{1}, \mathbf{p}_{2}\right)= & {\left[f^{*}\left(\mathbf{r}_{1}, \mathbf{r}_{2}, \mathbf{p}_{1}, \mathbf{p}_{2}\right)+1\right]\left[g^{\dagger}\left(\mathbf{r}_{1}, \mathbf{r}_{2}, \mathbf{p}_{1}, \mathbf{p}_{2}\right)\right.} \\
& \left.-c^{\dagger}\left(\mathbf{r}_{1}, \mathbf{r}_{2}, \mathbf{p}_{1}, \mathbf{p}_{2}\right)\right] \\
& +\exp \left[\beta v\left(\mathbf{r}_{1}, \mathbf{r}_{2}\right)\right] g\left(\mathbf{r}_{1}, \mathbf{r}_{2}\right) f^{\dagger}\left(\mathbf{r}_{1}, \mathbf{r}_{2}, \mathbf{p}_{1}, \mathbf{p}_{2}\right),
\end{aligned}
$$

or, for a homogeneous system,

$$
\begin{aligned}
g^{\dagger}\left(\mathbf{r}_{12}, \mathbf{p}_{1}, \mathbf{p}_{2}\right)= & {\left[f^{*}\left(\mathbf{r}_{12}, \mathbf{p}_{1}, \mathbf{p}_{2}\right)+1\right]\left[g^{\dagger}\left(\mathbf{r}_{12}, \mathbf{p}_{1}, \mathbf{p}_{2}\right)\right.} \\
& \left.-c^{\dagger}\left(\mathbf{r}_{12}, \mathbf{p}_{1}, \mathbf{p}_{2}\right)\right] \\
& +\exp \left[\beta v\left(\mathbf{r}_{12}\right)\right] g\left(\mathbf{r}_{12}\right) f^{\dagger}\left(\mathbf{r}_{12}, \mathbf{p}_{1}, \mathbf{p}_{2}\right) .
\end{aligned}
$$

Equation (6) joined with Eq. (9) or Eq. (8) joined with Eq.(10) give a closed set of equations for $g^{\dagger}\left(\mathbf{r}_{1}, \mathbf{r}_{2}, \mathbf{p}_{1}, \mathbf{p}_{2}\right)$.

From the function $g^{\dagger}\left(\mathbf{r}_{1}, \mathbf{r}_{2}, \mathbf{p}_{1}, \mathbf{p}_{2}\right)$ we define the pair correlation function for chemical clusters,

$$
g_{\text {chem }}\left(\mathbf{r}_{1}, \mathbf{r}_{2}\right)=\int \rho\left(\mathbf{r}_{1}, \mathbf{p}_{1}\right) \rho\left(\mathbf{r}_{2}, \mathbf{p}_{2}\right) g^{\dagger}\left(\mathbf{r}_{1}, \mathbf{r}_{2}, \mathbf{p}_{1}, \mathbf{p}_{2}\right) d \mathbf{p}_{1} d \mathbf{p}_{2} .
$$

This function is the joint probability density of finding two particles within the same chemical cluster at positions $\mathbf{r}_{1}$ and $\mathbf{r}_{2}$, respectively. Then the mean cluster size $S_{\text {chem }}$ and the percolation density $\rho_{p}$ are calculated as

$$
\begin{gathered}
S_{\text {chem }}(\rho)=1+\frac{1}{(N-1)} \int g_{\text {chem }}\left(\mathbf{r}_{1}, \mathbf{r}_{2}\right) d \mathbf{r}_{1} d \mathbf{r}_{2}, \\
\lim _{\rho \rightarrow \rho_{p}^{-}} S_{\text {chem }}(\rho)=\infty .
\end{gathered}
$$

\section{SOLUTION OF THE INTEGRAL EQUATION}

\section{A. Equivalence with an integral equation for polarizable fluids}

Our problem consists in solving Eq. (8) for $g^{\dagger}\left(\mathbf{r}_{12}, \mathbf{p}_{1}, \mathbf{p}_{2}\right)$ closed by the connectedness Percus-Yevick relation (10) with $f^{\dagger}\left(\mathbf{r}_{i}, \mathbf{r}_{j}, \mathbf{p}_{i}, \mathbf{p}_{j}\right)$ and $f^{*}\left(\mathbf{r}_{i}, \mathbf{r}_{j}, \mathbf{p}_{i}, \mathbf{p}_{j}\right)$ given by Eqs. (4) and (5). In the closure relation (10), $g\left(\mathbf{r}_{12}\right)$ is the thermal PDF of the system. We consider here a Lennard-Jones fluid whose particles interact through the pair potential 


$$
v(r)=4 \varepsilon\left[\left(\frac{\sigma}{r}\right)^{12}-\left(\frac{\sigma}{r}\right)^{6}\right] .
$$

In this work we take $g\left(\mathbf{r}_{12}\right)$ from the solution of the thermal Ornstein-Zernike (OZ) equation in the Percus-Yevick approximation [32].

An equation mathematically equivalent to Eq. (8) has been previously solved in II by one of us in the study of nonpolar polarizable molecules. Explicitly, the equation considered there, which is a generalized $\mathrm{OZ}$ equation, relates the fluid total correlation function (TCF) $h\left(\mathbf{r}_{12}, \mathbf{p}_{1}, \mathbf{p}_{2}\right)$ $=g\left(\mathbf{r}_{12}, \mathbf{p}_{1}, \mathbf{p}_{2}\right)-1\left[\right.$ with $g\left(\mathbf{r}_{12}, \mathbf{p}_{1}, \mathbf{p}_{2}\right)$ the PDF] and the direct correlation function (DCF) $c\left(\mathbf{r}_{12}, \mathbf{p}_{1}, \mathbf{p}_{2}\right)$,

$$
\begin{aligned}
h\left(\mathbf{r}_{12}, \mathbf{p}_{1}, \mathbf{p}_{2}\right)= & c\left(\mathbf{r}_{12}, \mathbf{p}_{1}, \mathbf{p}_{2}\right) \\
& +\rho \int f\left(p_{3}\right) c\left(\mathbf{r}_{13}, \mathbf{p}_{1}, \mathbf{p}_{3}\right) h\left(\mathbf{r}_{32}, \mathbf{p}_{3}, \mathbf{p}_{2}\right) d \mathbf{r}_{3} d \mathbf{p}_{3},
\end{aligned}
$$

where $\mathbf{p}_{i}$ denotes the instantaneous dipolar moment induced on molecule $i$ by the remaining molecules of the system. The function $f(p)$ gives the instantaneous dipolar moment thermal distribution which, in II, is assumed to have a Gaussian form

$$
f(p)=\frac{1}{(2 \pi \alpha / \beta)^{3 / 2}} \exp \left(-\frac{\beta p^{2}}{2 \alpha}\right),
$$

where $\alpha$ is the effective polarizability of the molecules.

We observe that Eqs. (8) and (15) are the same equation if we identify $h$ with $g^{\dagger}, c$ with $c^{\dagger}$, the induced dipolar moment $\mathbf{p}_{i}$ with the kinetic momentum $\mathbf{p}_{i}$ and the polarizability $\alpha$ with the particle mass $m$. There are, however, some differences between the connectivity problem and the polarizablemolecule problem described in II. The form of $f(p)$ does not need to be Gaussian in II; moreover, $f(p)$ is coupled to the TCF. Therefore, the value of the effective polarizability $\alpha$ depends on the density and temperature of the system. In the connectivity problem, however, the equivalent of $f(p)$, $\rho(\mathbf{r}, \mathbf{p}) / \rho$, is intrinsically Gaussian and independent of the thermodynamic macrostate of the system.

Another difference between the connectivity problem here and the problem described in II is that our closure relation must be complemented with the condition given by Eq. (4). This means that we have to calculate the path of a given pair of molecules over a period of time $\tau$. In addition, the closures are different. Here we consider the connectedness version of Percus-Yevick whereas an almost exact relation between DCF and TCF (van Leeuwen-Groeneveld-De Boer [35] exact relation with approximate bridge function) is used in II. Nevertheless, these differences do not affect the general method of solution developed in II and we can apply the same principle of expansions in orthogonal functions.

Thus, following II, we start by reassigning the unknown function to be the indirect correlation function

$$
\gamma^{\dagger}\left(\mathbf{r}_{12}, \mathbf{p}_{1}, \mathbf{p}_{2}\right)=g^{\dagger}\left(\mathbf{r}_{12}, \mathbf{p}_{1}, \mathbf{p}_{2}\right)-c^{\dagger}\left(\mathbf{r}_{12}, \mathbf{p}_{1}, \mathbf{p}_{2}\right),
$$

rather than $g^{\dagger}\left(\mathbf{r}_{12}, \mathbf{p}_{1}, \mathbf{p}_{2}\right)$, and rewriting Eq. (8) in Fourier transform representation,

$$
\begin{aligned}
\tilde{\gamma}^{\dagger}\left(\mathbf{k}, \mathbf{p}_{1}, \mathbf{p}_{2}\right)= & \frac{\rho}{\left(2 \pi m k_{B} T\right)^{3 / 2}} \int d \mathbf{p}_{3} \exp \left[-\beta \frac{p_{3}^{2}}{2 m}\right]\left[\tilde{\gamma}^{\dagger}\left(\mathbf{k}, \mathbf{p}_{1}, \mathbf{p}_{3}\right)\right. \\
& \left.+\tilde{c}^{\dagger}\left(\mathbf{k}, \mathbf{p}_{1}, \mathbf{p}_{3}\right)\right] \widetilde{c}^{\dagger}\left(\mathbf{k}, \mathbf{p}_{3}, \mathbf{p}_{2}\right) .
\end{aligned}
$$

The closure given by the Percus-Yevick relation [Eq. (10)] together with the conditions (4) and (5) yield

$$
\begin{aligned}
& c^{\dagger}\left(\mathbf{r}_{12}, \mathbf{p}_{1}, \mathbf{p}_{2}\right) \\
& \quad=\left\{\begin{array}{l}
g\left(\mathbf{r}_{12}\right)-\gamma^{\dagger}\left(\mathbf{r}_{12}, \mathbf{p}_{1}, \mathbf{p}_{2}\right), \quad\left|\mathbf{r}_{12}(t)\right|<d \forall t \leqslant \tau, \\
\left(\exp \left[-\beta v\left(\mathbf{r}_{12}\right)\right]-1\right) \gamma^{\dagger}\left(\mathbf{r}_{12}, \mathbf{p}_{1}, \mathbf{p}_{2}\right), \quad \text { otherwise. }
\end{array}\right.
\end{aligned}
$$

The connectivity part of the PDF is then computed from $\gamma^{\dagger}$ as

$$
g^{\dagger}\left(\mathbf{r}_{12}, \mathbf{p}_{1}, \mathbf{p}_{2}\right)=\left\{\begin{array}{l}
g\left(\mathbf{r}_{12}\right) \quad\left|\mathbf{r}_{12}(t)\right|<d \forall t \leqslant \tau, \\
\exp \left[-\beta v\left(\mathbf{r}_{12}\right)\right] \gamma^{\dagger}\left(\mathbf{r}_{12}, \mathbf{p}_{1}, \mathbf{p}_{2}\right), \quad \text { otherwise. }
\end{array}\right.
$$

The Fourier transform in Eq. (18) and its inverse are defined as

$$
\begin{gathered}
\tilde{f}(\mathbf{k})=\int d \mathbf{r} f(\mathbf{r}) e^{-i \mathbf{k} \cdot \mathbf{r}}, \\
f(\mathbf{r})=\frac{1}{(2 \pi)^{3}} \int d \mathbf{k} \tilde{f}(\mathbf{k}) e^{i \mathbf{k} \cdot \mathbf{r}} .
\end{gathered}
$$

The standard method for solving Eqs. (18) and (19) is to explicitly break out the angular dependence of all functions in the form of expansions in spherical harmonics [36]. The general expansions for pair functions in real as well as in transformed spaces are shown in the Appendix.

Introducing the expansion for $\tilde{\gamma}^{\dagger}\left(\mathbf{k}, \mathbf{p}_{1}, \mathbf{p}_{2}\right)$ and the corresponding expansion for $\widetilde{c}^{\dagger}\left(\mathbf{k}, \mathbf{p}_{1}, \mathbf{p}_{2}\right)$, one finds that the OZlike equation in Fourier space [Eq. (18)] goes over into a set of matrix equations for the respective coefficients,

$$
\tilde{\gamma}_{l_{1} l_{2} m}^{\dagger n_{1} n_{2}}(k)=(-1)^{m} \rho \sum_{n_{3}, l_{3}}\left[\tilde{\gamma}_{l_{1} l_{3} m}^{\dagger n_{1} n_{3}}(k)+\tilde{c}_{l_{1} l_{3} m}^{\dagger n_{1} n_{3}}(k)\right] \tilde{c}_{l_{3} l_{2} m}^{\dagger n_{3} n_{2}}(k) .
$$

\section{B. Numerical procedure}

To obtain a numerical solution for the set of equations (8) and (10) one needs the discrete versions of the expansion for $\gamma^{\dagger}\left(\mathbf{r}, \mathbf{p}_{1}, \mathbf{p}_{2}\right)$ [Eq. (A9)] and the quadratures for the coefficients $\gamma_{l_{1} l_{2} m}^{\dagger n_{1} n_{2}}(r)$ [Eq. (A11)]; these are

$$
\begin{aligned}
\gamma^{\dagger}\left(r, i_{1}, i_{2}, k_{1}, k_{2}, j\right)= & 4 \pi \sum_{n_{1}, n_{2}, l_{1}, l_{2}, m} \gamma_{l_{1} l_{2} m}^{\dagger n_{1} n_{2}}(r) Q_{n_{1} l_{1}}\left(i_{1}\right) Q_{n_{2} l_{2}}\left(i_{2}\right) \\
& \times \mathcal{P}_{l_{1} m}\left(k_{1}\right) \mathcal{P}_{l_{2} m}\left(k_{2}\right) \nu_{m} T_{m}(j)
\end{aligned}
$$

and 


$$
\begin{aligned}
\gamma_{l_{1} l_{2} m}^{\dagger n_{1} n_{2}}(r)= & \sum_{i_{1}, i_{2}, k_{1}, k_{2}, j=1}^{N_{p}} w\left(i_{1}\right) w\left(i_{2}\right) w\left(k_{1}\right) w\left(k_{2}\right) w(j) \\
& \times \gamma^{\dagger}\left(r, i_{1}, i_{2}, k_{1}, k_{2}, j\right) Q_{n_{1} l_{1}}\left(i_{1}\right) Q_{n_{2} l_{2}}\left(i_{2}\right) \\
& \times \mathcal{P}_{l_{1} m}\left(k_{1}\right) \mathcal{P}_{l_{2} m}\left(k_{2}\right)(-1)^{m} T_{m}(j) .
\end{aligned}
$$

In Eq. (24), $\nu_{0}=1$ and $\nu_{m}=2$ for $m>0$. In Eq. (25), Gaussian quadratures are being used, with the argument $i$ standing for $t_{i}=\beta p_{i}^{2} / 2 m$, the $i$ th root of $L_{N_{p}}^{1 / 2}(t), k$ for $x_{k}=\cos \theta_{k}$, the $k$ th root of $P_{N_{p}}(x)$, and $j$ for $y_{j}=\cos \phi_{j}$, the $j$ th root of $T_{N_{p}}(y)$, where $L_{N_{p}}^{1 / 2 p}(t), P_{N_{p}}(x)$, and $T_{N_{p}}(y)$ are the associated Laguerre, Legendre, and Chebyshev polynomials, respectively, all of order $N_{p}$; here the associated Legendre functions $\mathcal{P}_{l m}(x)$ are normalized to 2. The $w$ are the corresponding Gaussian weights,

$$
\begin{gathered}
w(i)=\left\{t_{i}\left[L_{N_{p}}^{1 / 2^{\prime}}\left(t_{i}\right)\right]^{2}\right\}^{-1}, \\
w(k)=\left\{\left(1-x_{k}^{2}\right)\left[P_{N_{p}}^{\prime}\left(x_{k}\right)\right]^{2}\right\}^{-1}, \\
w(j)=N_{p}^{-1},
\end{gathered}
$$

where the prime denotes derivative.

The solution follows an iterative procedure. The preparatory stages of the calculation consist of (i) computing the thermal PDF $g\left(r_{12}\right)$ for the Lennard-Jones fluid over a suitable mesh using the Percus-Yevick equation, (ii) reducing the momentum space to the discrete set of points $\mathbf{p}_{i, k, j}$ $\equiv\left(p_{i}, \theta_{k}, \phi_{j}\right)$ with $i, k, j=1,2, \ldots, N_{p}$, and (iii) identifying the subset of states -within all possible configurational states $\left(r_{12}, \mathbf{p}_{1}, \mathbf{p}_{2}\right)$ of a pair of particles - that correspond to a bonded pair.

The third preparatory step above is carried out as follows. The relative distance $r_{12}$ between particles 1 and 2 and the momenta $\mathbf{p}_{1 ; i, k, j} \equiv\left(p_{1 i}, \theta_{1 k}, \phi_{1 j}\right.$ and $\mathbf{p}_{2 ; i, k, j} \equiv\left(p_{2 i}, \theta_{2 k}, \phi_{2 j}\right)$ are made to run over all the mesh values and the reduced momentum space [step (ii)], respectively. Thus, for each set $\left(r_{12}, \mathbf{p}_{1 ; i, k, j}, \mathbf{p}_{2 ; i, k, j}\right)$ taken as initial condition, we consider the equation of motion of the two particles

$$
\frac{m}{2} \frac{\partial^{2} r(t)}{\partial t^{2}}=-\frac{\partial v^{\mathrm{eff}}[r(t)]}{\partial r}+\frac{L^{2}}{\frac{m}{2}[r(t)]^{3}},
$$

where $r(t)=\left|\mathbf{r}_{1}(t)-\mathbf{r}_{2}(t)\right|, \quad v^{\operatorname{eff}}[r(t)]=-k_{B} T \ln g[r(t)]$, and $L$ $=|\mathbf{L}|=|\mathbf{r}(t) \times \mathbf{p}(t)|=$ const. In the last equality we have $\mathbf{r}(t)$ $=\mathbf{r}_{1}(t)-\mathbf{r}_{2}(t)$ and $\mathbf{p}(t)=\mathbf{p}_{1}(t)-\mathbf{p}_{2}(t)$. Equation (29) must be solved with the initial conditions

$$
r(t)=\left|\mathbf{r}_{1}(t)-\mathbf{r}_{2}(t)\right|_{t=0}=\left|\mathbf{r}_{1}-\mathbf{r}_{2}\right|=r_{12}
$$

and

$$
\begin{aligned}
\left.\frac{\partial r(t)}{\partial t}\right|_{t=0} & =\left|\dot{\mathbf{r}}_{1}(t)-\dot{\mathbf{r}}_{2}(t)\right|_{t=0}=\left|\frac{\mathbf{p}_{1}(t)}{m}-\frac{\mathbf{p}_{2}(t)}{m}\right|_{t=0} \\
& =\left|\frac{\mathbf{p}_{1}}{m}-\frac{\mathbf{p}_{2}}{m}\right|=\frac{p_{12}}{m} .
\end{aligned}
$$

Thus, for each set of values $\left(r_{12}, \mathbf{p}_{1}, \mathbf{p}_{2}\right)$ we must solve the differential equation (29) with the initial conditions given by Eqs. (30) and (31). This problem can be put in a more adequate form to be solved using the Runge-Kutta method:

$$
\begin{gathered}
\frac{d r(t)}{d t}=s(t), \\
\frac{d s(t)}{d t}=f[r(t)], \\
\left.r(t)\right|_{t=0}=r_{12}, \\
\left.s(t)\right|_{t=0}=\left.\frac{d r(t)}{d t}\right|_{t=0}=\frac{p_{12}}{m}, \\
\left.\frac{d s(t)}{d t}\right|_{t=0}=f[r(t=0)],
\end{gathered}
$$

where

$$
f(r)=\frac{1}{\frac{m}{2} \beta g(r)} \frac{d g(r)}{d r}+\frac{\left(r_{12} p_{12}\right)^{2}\left(1-x_{12}^{2}\right)}{\left(\frac{m}{2}\right)^{2} r^{3}} .
$$

From the numerical solution of the equation of motion, we construct a logical array $B\left(r_{12}, \mathbf{p}_{1 ; i, k, j}, \mathbf{p}_{2 ; i, k, j}\right)$ of dimension seven whose value is TRUE if the configurational state of the pair of particles corresponds to a bonded state, i.e., if $r_{12}(t)$ $<d \forall t \leqslant \tau$. If instead the condition $r_{12}(t)<d \forall t \leqslant \tau$ is not satisfied, then $B\left(r_{12}, \mathbf{p}_{1 ; i, k, j}, \mathbf{p}_{2 ; i, k, j}\right)$ is FALSE.

The iterative solution of Eqs. (23) and (19) starts by guessing the initial values of the coefficients $\gamma_{l_{1} l_{2} m}^{n_{1} n_{2}}\left(r_{12}\right)$. Then, if $B\left(r_{12}, \mathbf{p}_{1 ; i, k, j}, \mathbf{p}_{2 ; i, k, j}\right)$ is TRUE, following Eq. (20) we take

$$
g_{l_{1} l_{2} m}^{\dagger n_{1} n_{2}}\left(r_{12}\right)=\left\{\begin{array}{l}
g\left(r_{12}\right), \quad \text { if } n_{1}=n_{2}=l_{1}=l_{2}=m=0, \\
0, \quad \text { otherwise. }
\end{array}\right.
$$

If instead $B\left(r_{12}, \mathbf{p}_{1 ; i, k, j}, \mathbf{p}_{2 ; i, k, j}\right)$ is FALSE then, following Eq. (20), we take

$$
g_{l_{1} l_{2} m}^{\dagger n_{1} n_{2}}\left(r_{12}\right)=\exp \left[-\beta v\left(r_{12}\right)\right] \gamma_{l_{1} l_{2} m}^{\dagger n_{1} n_{2}}\left(r_{12}\right) .
$$

Knowing $g_{l_{1} l_{2} m}^{\dagger n_{1} n_{2}}\left(r_{12}\right)$ and $\gamma_{l_{1} l_{2} m}^{n_{1} n_{2}}\left(r_{12}\right)$ for all the mesh points and allowed indices, we can calculate [see Eqs. (17) or (19)]

$$
c_{l_{1} l_{2} m}^{\dagger n_{1} n_{2}}\left(r_{12}\right)=g_{l_{1} l_{2} m}^{\dagger n_{1} n_{2}}\left(r_{12}\right)-\gamma_{l_{1} l_{2} m}^{\dagger n_{1} n_{2}}\left(r_{12}\right) \text {. }
$$

We now need to transform the coefficients $c_{l_{1} l_{2} m}^{n_{1} n_{2}}\left(r_{12}\right)$ in real space into coefficients $\widetilde{c}_{l_{1} l_{2} m}^{\dagger n_{1} n_{2}}(k)$ in Fourier space. However, as we have mentioned, they are not themselves Fourier transforms of each other. Thus, we have to assemble the complete function first using the equation analogous to Eq. (24) for $c^{\dagger}\left(r, i_{1}, i_{2}, k_{1}, k_{2}, j\right)$ and then use a generalized fasttransform algorithm [33] to calculate $\widetilde{c}^{\dagger}\left(k, i_{1}, i_{2}, k_{1}, k_{2}, j\right)$. Using the equation analogous to Eq. (25) in $\mathbf{k}$ space we then have the coefficients $\widetilde{c}_{l_{1} l_{2} m}^{i n} n_{1} n_{2}(k)$ for the complete set of indices 


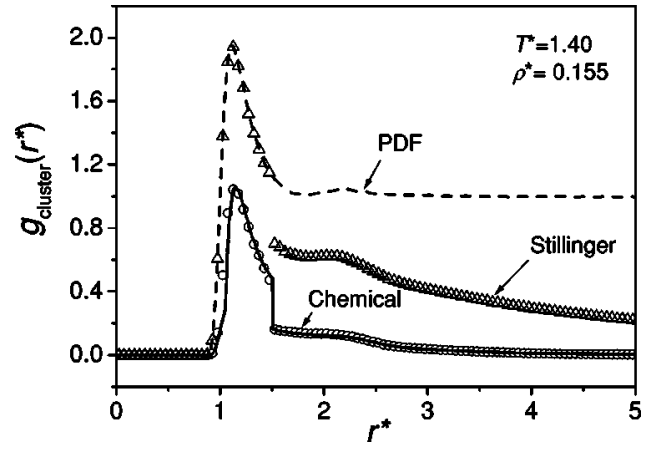

FIG. 1. Cluster pair correlation function. Full line and circles correspond to chemical clusters with $d^{*}=1.5$ and $\tau^{*}=0.5$ as obtained from the integral equation and $\mathrm{MD}$, respectively. Triangles correspond to Stillinger clusters $\left(\tau^{*}=0\right)$ with $d^{*}=1.5$ as obtained from MD. Dashed line is the thermal PDF as obtained from MD. The system is at $T^{*}=1.4$ and $\rho^{*}=0.155$.

and all the values of $k$ on an adequate mesh. The coefficients $\tilde{\gamma}_{l_{1} l_{2}}^{n_{1} n_{2}}(k)$ are then easily calculated by using the OZ-like equation in Fourier space [see Eq. (23)]. Again we assemble the complete function $\tilde{\gamma}^{\dagger}\left(k, i_{1}, i_{2}, k_{1}, k_{2}, j\right)$ [using the Fourier space version of Eq. (24)]. The inverse transform $\gamma^{\dagger}\left(r_{12}, i_{1}, i_{2}, k_{1}, k_{2}, j\right)$ is calculated with the fast-transform algorithm and so new coefficients $\gamma_{l_{1} l_{2} m}^{n_{1} n_{2}}\left(r_{12}\right)$ [obtained from Eq. (25)] are again available to reinitiate the iterative cycle. The iterations end when convergence is reached, as measured by

$$
\left|\left[\gamma_{l_{1} l_{2} m}^{\dagger n_{1} n_{2}}\left(r_{12}\right)\right]_{(s+1) \text { th iteration }}-\left[\gamma_{l_{1} l_{2} m}^{\dagger n_{1} n_{2}}\left(r_{12}\right)\right]_{\text {sth iteration }}\right|<\epsilon
$$

for the complete set of indices. The tolerance $\epsilon$ is set to 0.0001 .

The pair correlation function for a chemical cluster [see Eq. (11)] is finally given by

$$
g_{\text {chem }}\left(r_{12}\right)=g_{000}^{\dagger 00}\left(r_{12}\right),
$$

where the orthonormality condition [see Eq. (A12)] has been used.

\section{RESULTS AND DISCUSSION}

Following the method of the previous section, we have solved the integral equation (8) for a Lennard-Jones fluid with a pair potential given by Eq. (14) and we have calculated the corresponding $g_{\text {chem }}\left(r_{12}\right)$ according to Eq. (38). In order to check the theory, MD simulations on the same system have been performed; the simulation details are given elsewhere [20]. All the quantities reported here are in reduced units: $r^{*}=r / \sigma, \rho^{*}=\rho \sigma^{3}, T^{*}=k_{B} T / \varepsilon, d^{*}=d / \sigma$ and $\tau^{*}$ $=\tau \sigma^{-1} \sqrt{\varepsilon / m}$ for the density, temperature, connectivity distance, and residence lifetime, respectively. For the results shown below we have used $d^{*}=1.5$ and $\tau^{*}=0.5$.

In Figs. 1-3 we show the theoretical cluster correlation functions $g_{\text {chem }}\left(r_{12}\right)$ calculated for $\rho^{*}=0.155, T^{*}=1.4 ; \rho^{*}$ $=0.155, T^{*}=2.0$; and $\rho^{*}=0.26, T^{*}=2.0$, along with the corre-

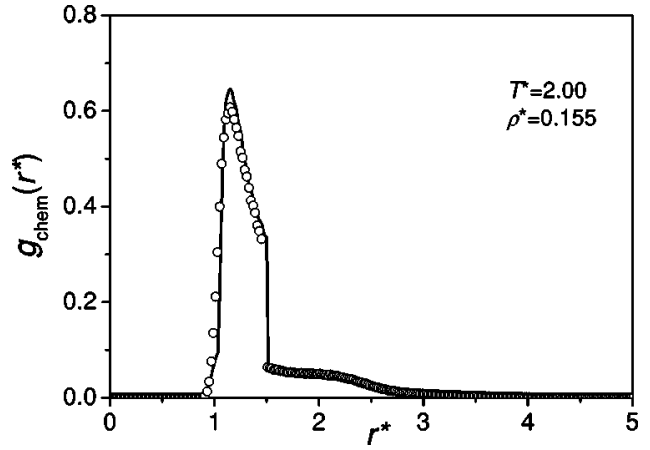

FIG. 2. The pair correlation functions for chemical clusters with $d^{*}=1.5$ and $\tau^{*}=0.5$ at $T^{*}=2.0$ and $\rho^{*}=0.155$. Results from the integral equation theory (full line) and from MD simulation (circles) are shown.

sponding simulation results. In Fig. 1 we also show the cluster correlation function $g_{\mathrm{Still}}\left(r_{12}\right)$ obtained from molecular dynamics when the Stillinger connectivity criterion, namely when $\tau^{*}=0$, is used instead. In addition, the thermal PDF $g\left(r_{12}\right)$ from MD is shown as a guide. It should be mentioned that the percolation density $\rho_{p}^{*}$ for our chemical clusters is about 0.26 for $T^{*}=1.4$ and 0.32 for $T^{*}=2.0$ according to our simulations. The density $\rho^{*}=0.155$ corresponds to the percolation density for Stillinger clusters at $T^{*}=1.4$.

The qualitative behavior of these curves agrees with the general trends encountered in connectedness studies in continuum systems. The discontinuity at $r_{12}=d$ for Stillinger (see Fig. 1) as well as for chemical clusters is a typical feature: the probability for two particles to be connected at $r_{12}$ $>d$, even for $r_{12} \rightarrow d^{+}$, depends on the presence of an intermediate third particle directly connected to the other two and thus the probability of belonging to the cluster notably decreases. For clusters defined according to the Stillinger criterion $(\tau=0)$, two particles which are separated by a distance shorter than the connectivity distance belong to the same Stillinger cluster with certainty. Thus, for $r_{12}<d$, the function $g_{\text {Still }}\left(r_{12}\right)$ coincides with the ordinary PDF $g\left(r_{12}\right)$. As expected, the probability density of finding two particles connected according to the chemical criterion $(\tau \neq 0)$ is smaller than $g_{\text {Still }}\left(r_{12}\right)$ for any $r_{12}$. This is to be expected

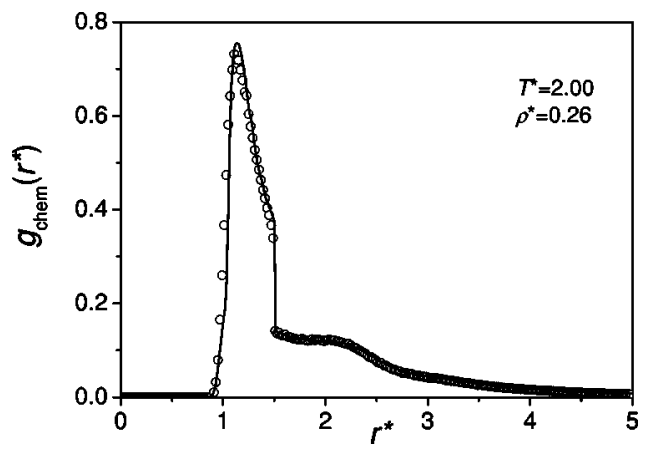

FIG. 3. The pair correlation functions for chemical clusters with $d^{*}=1.5$ and $\tau^{*}=0.5$ at $T^{*}=2.0$ and $\rho^{*}=0.26$. Results from the integral equation theory (full line) and from MD simulation (circles) are shown. 


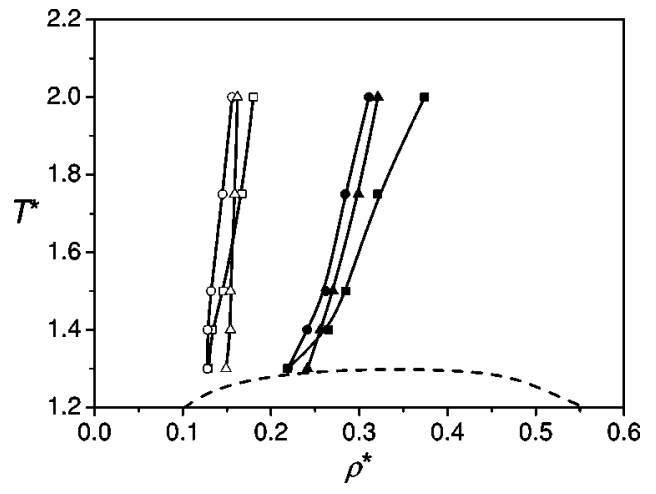

FIG. 4. The percolation loci for clusters with $d^{*}=1.5$. The empty symbols correspond to Stillinger clusters $\left(\tau^{*}=0\right)$ and the full symbols to chemical clusters with $\tau^{*}=0.5$. Circles and squares were calculated from the theory using the power law [Eq. (39)] with $\gamma$ variable and $\gamma=2$, respectively. Triangles were obtained from MD. The full lines are only a guide to the eye. The dashed line is an interpolation to the Monte Carlo coexistence curve obtained by $\mathrm{Pa}$ nagiotopoulos (Ref. [39]).

since a dynamic restriction is required in addition to the geometrical one. In particular, for $r_{12}<d, g_{\text {chem }}\left(r_{12}\right)$ is always smaller than the thermal PDF. The function $g_{\text {Still }}\left(r_{12}\right)$ in Fig. 1 is long ranged because, as mentioned above, the density considered in that case corresponds to the percolation density for Stillinger clusters at the given temperature. The mean cluster size $S_{\text {Still }}$ diverges at that density.

From these figures we can conclude that, at least for the densities and temperatures considered, the integral equation results reproduce rather well those obtained from MD simulations.

In Fig. 4 we present the gas-liquid coexistence curve in the $T-\rho$ plane obtained by Panagiotopoulos [39], using Gibbs ensemble Monte Carlo simulations, together with the percolation loci for the chemical clusters $(\tau=0.5)$ and Stillinger clusters $(\tau=0)$. These percolation curves separate the phase diagram in two parts: percolated (high densities) and nonpercolated (low densities). They were calculated from our theory using Eqs. (12) and (13), and also from MD (see Ref. [20] for details).

To calculate the critical density $\rho_{\mathrm{p}}$ for a given temperature we use the critical power law

$$
S_{\text {chem }}(\rho) \sim\left|\rho-\rho_{\mathrm{p}}\right|^{-\gamma}
$$

as an extrapolation formula. The fitting was performed in two alternative ways: (i) fixing the exponent at the value $\gamma$ $=2$, and (ii) allowing $\gamma$ to vary freely. The value $\gamma=2$ is the known mean field critical exponent obtained from the Percus-Yevick approximation when only a geometric restriction is required in the cluster definition (which is the case of the Stillinger clusters [40]). The critical exponent $\gamma$ for Stillinger clusters in Lennard-Jones fluids, obtained from MD simulations, was reported by Heyes and Melrose [41] as being near the universal value $\gamma=1.8$ observed in lattice systems. In Fig. 4, the theoretical percolation curves for $\tau=0.5$ and $\tau=0$ calculated by using the two fitting procedures and that obtained from MD can be seen. For $\tau=0.5$ we clearly observe that the theoretical curve extrapolated using $\gamma$ variable agrees with the simulation data better than the curve obtained by using $\gamma \equiv 2$, although the theoretical prediction is slightly shifted to lower densities with respect to the MD prediction. For Stillinger clusters, things are less clear at first sight. However, if we calculate the mean quadratic error, $\Delta \rho_{\mathrm{p}}^{*}=\sqrt{\sum_{T^{*}}\left(\rho_{\text {theor }}^{*}-\rho_{\text {simul }}^{*}\right)^{2}}$, we observe that $\left[\Delta \rho_{\mathrm{p}}^{*}\right]_{\gamma=2}$ $<\left[\Delta \rho_{\mathrm{p}}^{*}\right]_{\gamma}$ variable, which suggest that the agreement with the MD percolation loci is better when a constant exponent 2 is used.

From Fig. 4 we can observe that the predictability of the theory is as good for $\tau \neq 0$ as for $\tau=0$. However, for values of $\tau$ much larger than the one shown here, the corresponding percolation density will be significantly higher and the approximation introduced by the potential of mean force in the equation of motion will eventually fail. It is worth mentioning that the deviations observed between MD and the theoretical results are not entirely due to the approximations introduced in the numerical solution of Eq. (8). In practice, in a MD simulation [20], the percolation density is calculated as the density at which 50 percent of the configurations present a percolating cluster. This technique is rather different to the extrapolation described above for the theoretical prediction of the percolation density.

\section{CONCLUSIONS}

We have numerically solved a connectedness integral equation theory that describes clusters where the particleparticle bonds are identified by setting a connectivity distance $d$ plus a lifespan $\tau$. The technique used consists in expanding the pair correlation functions in orthogonal polynomials. These same polynomials were considered originally in the study of the "thermal" correlation function of polarizable molecules. The numerical results within the PercusYevick approximation that are obtained here agree rather well with earlier MD simulations, at least at the densities studied, in the case of Lennard-Jones particles.

The use of an explicit lifetime for the bonding criterion is especially important when one tries to compare cluster properties extracted from the theory with those measured in experiments. The sol-gel transition in weakly attractive colloids, for example, is associated with the percolation of the clusters due to weak colloidal interactions. However, if the transition is measured by linear viscoelastic rheology, the position of the sol-gel line depends on the testing frequency [42]. High frequencies are able to detect only very shortlived bonds and clusters whereas low frequencies detect more long-lasting bonds and clusters. A discussion on the relationship between this frequency effect and the chemical clusters considered in this paper can be found in Ref. [30].

Since MD is a very costly technique to study long lasting clusters [20], we expect that our numerical approach will be particularly useful to estimate the percolation line for moderately large values of $\tau$. Large values of $\tau$ are of particular interest since they lead to the identification of more stable clusters that are easier to detect in experiments.

\section{ACKNOWLEDGMENTS}

We are grateful to Ileana F. Márquez for her help in the adaptation of the algorithms. This work was supported by 
CONICET, CICPBA, ANPCyT and UNLP (Argentina), and CSIC (Spain). E.L. acknowledges support from the Dirección General de Universidades e Investigación Científica under Grant BFM2001-1017-C03-01.

\section{APPENDIX: EXPANSION OF THE PAIR FUNCTIONS IN ORTHOGONAL POLYNOMIALS}

The essential point in the integral equation solution method [33] is the expansion of all the pair functions, like $\gamma^{\dagger}\left(\mathbf{r}_{12}, \mathbf{p}_{1}, \mathbf{p}_{2}\right)$, in terms of orthogonal polynomials. Here we sketch how these expansions follow. First we expand:

$$
\begin{aligned}
\gamma^{\dagger}\left(\mathbf{r}_{12}, \mathbf{p}_{1}, \mathbf{p}_{2}\right) & =\gamma^{\dagger}\left(r, p_{1}, p_{2}, \omega_{1}, \omega_{2}\right) \\
& =4 \pi \sum_{l_{1}, l_{2}, m} \gamma_{l_{1} l_{2} m}^{\dagger}\left(r, p_{1}, p_{2}\right) Y_{l_{1} m}\left(\omega_{1}\right) Y_{l_{2} \bar{m}}\left(\omega_{2}\right),
\end{aligned}
$$

where $\omega_{1} \omega_{2}$, are the directions of the momenta $\mathbf{p}_{1}$ and $\mathbf{p}_{2}$, $\bar{m}=-m$, and $m=-l,-l+1, \ldots, l$. In this and similar expressions, the vector $\mathbf{r}_{12}$ has been implicitly chosen as the $z$ direction in the specification of the Euler angles $\omega=(\theta, \phi)$. The spherical harmonics satisfy the orthogonality condition

$$
\int d \omega Y_{l m}(\omega) Y_{l^{\prime} m^{\prime}}^{*}(\omega)=\delta_{l l^{\prime}} \delta_{m m^{\prime}}
$$

so that the coefficients of the expansion (A1) are immediately obtainable as

$$
\begin{aligned}
\gamma_{l_{1} l_{2} m}^{\dagger}\left(r, p_{1}, p_{2}\right)= & \frac{1}{4 \pi} \int d \omega_{1} d \omega_{2} \gamma^{\dagger}\left(r, p_{1}, p_{2}, \omega_{1}, \omega_{2}\right) \\
& \times Y_{l_{1} m}\left(\omega_{1}\right) Y_{l_{2} m}^{*}\left(\omega_{2}\right) .
\end{aligned}
$$

Similarly, we can break out the kinetic momentum dependence in the form of expansions in polynomials of $p$,

$$
\gamma_{l_{1} l_{2} m}^{\dagger}\left(r, p_{1}, p_{2}\right)=\sum_{n_{1}, n_{2}} \gamma_{l_{1} l_{2} m}^{\dagger n_{1} n_{2}}(r) Q_{n_{1} l_{1}}\left(p_{1}\right) Q_{n_{2} l_{2}}\left(p_{2}\right)
$$

which are constructed to be orthogonal with Gaussian weight function

$$
f(p)=\frac{1}{(2 \pi m / \beta)^{3 / 2}} \exp \left[-\beta p^{2} / 2 m\right]
$$

namely,

$$
4 \pi \int_{0}^{\infty} d p p^{2} f(p) Q_{n l}(p) Q_{n^{\prime} l}(p)=\delta_{n n^{\prime}}
$$

The coefficients of the expansion are then again obtainable by quadratures,

$$
\begin{aligned}
\gamma_{l_{1} l_{2} m}^{\dagger n_{1} n_{2}}(r)= & \int_{0}^{\infty} d p_{1} d p_{2}\left[4 \pi p_{1}^{2} f\left(p_{1}\right)\right]\left[4 \pi p_{2}^{2} f\left(p_{2}\right)\right] \\
& \times \gamma_{l_{1} l_{2} m}^{\dagger}\left(r, p_{1}, p_{2}\right) Q_{n_{1} l_{1}}\left(p_{1}\right) Q_{n_{2} l_{2}}\left(p_{2}\right) .
\end{aligned}
$$

Given the Gaussian form of the weight function $f(p)$, the associated polynomials are [37]

$$
Q_{n l}(p)=\left[\frac{\Gamma\left(\frac{1}{2}(n-l)+1\right) \Gamma\left(\frac{3}{2}\right)}{\Gamma\left(\frac{1}{2}(n+l)+\frac{3}{2}\right)}\right]^{1 / 2}\left(\frac{\beta p^{2}}{2 m}\right)^{l / 2} L_{(n-l) / 2}^{l+1 / 2}\left(\frac{\beta p^{2}}{2 m}\right),
$$

where $L_{n}^{b}(t)$ is an associated Laguerre polynomial [38] and $\Gamma(z)$ is the gamma function.

Accordingly, all the functions in $\mathbf{r}$ space are expanded in the form

$$
\begin{aligned}
\gamma^{\dagger}\left(\mathbf{r}, \mathbf{p}_{1}, \mathbf{p}_{2}\right)= & 4 \pi \sum_{n_{1}, n_{2}, l_{1}, l_{2}, m} \gamma_{l_{1} l_{2} m}^{\dagger n_{1} n_{2}}(r) Q_{n_{1} l_{1}}\left(p_{1}\right) Q_{n_{2} l_{2}}\left(p_{2}\right) \\
& \times Y_{l_{1} m}\left(\omega_{1}\right) Y_{l_{2} \bar{m}}\left(\omega_{2}\right),
\end{aligned}
$$

where the $z$ axis is along $\mathbf{r}$ and the summation indices satisfy the constraints

$$
\begin{gathered}
n=0,1,2, \ldots, \\
l=n, n-2, n-4, \ldots, 1 \text { or } 0, \\
m=0, \pm 1, \pm 2, \ldots, \pm l .
\end{gathered}
$$

The coefficients of Eq. (A9) can be obtained as

$$
\begin{aligned}
\gamma_{l_{1} l_{2} m}^{\dagger n_{1} n_{2}}(r)= & 4 \pi \int d \mathbf{p}_{1} d \mathbf{p}_{2} f\left(p_{1}\right) f\left(p_{2}\right) \gamma^{\dagger}\left(\mathbf{r}, \mathbf{p}_{1}, \mathbf{p}_{2}\right) \\
& \times Q_{n_{1} l_{1}}\left(p_{1}\right) Q_{n_{2} l_{2}}\left(p_{2}\right) Y_{l_{1} m}^{*}\left(\omega_{1}\right) Y_{l_{2} \bar{m}}^{*}\left(\omega_{2}\right)
\end{aligned}
$$

with $f(p)$ given by Eq. (A5). The complete orthonormality condition is

$$
4 \pi \int d \mathbf{p} f(p) Q_{n l}(p) Q_{n^{\prime} l^{\prime}}(p) Y_{l m}(\omega) Y_{l^{\prime} m^{\prime}}^{*}(\omega)=\delta_{n n^{\prime}} \delta_{l l^{\prime}} \delta_{m m^{\prime}}
$$

The functions in $\mathbf{k}$ are expandable in a similar way. Setting the $z$ axis along $\mathbf{k}$, we write

$$
\begin{aligned}
\tilde{\gamma}^{\dagger}\left(\mathbf{k}, \mathbf{p}_{1}, \mathbf{p}_{2}\right)= & 4 \pi \sum_{n_{1}, n_{2}, l_{1}, l_{2}, m}^{\dagger n_{1} n_{2}} \tilde{\gamma}_{l_{1} l_{2} m}^{\dagger n_{1} n_{2}}(k) Q_{n_{1} l_{1}}\left(p_{1}\right) Q_{n_{2} l_{2}}\left(p_{2}\right) \\
& \times Y_{l_{1} m}\left(\omega_{1}\right) Y_{l_{2} \bar{m}}\left(\omega_{2}\right) .
\end{aligned}
$$

However, the angles $\omega_{1}, \omega_{2}$ are referred to different axes in Eqs. (A9) and (A13), so that the coefficients in these expansions are not themselves mutual Fourier transforms. 
[1] S. H. Simon, V. Dobrodavjević, and R. M. Stratt, J. Chem. Phys. 94, 7360 (1991).

[2] G. S. Grest and M. H. Cohen, in Percolation Structures and Processes, edited by G. Deutscher, R. Zallen, and J. Adler (Adam Hilger, Bristol, 1983).

[3] H.-P. Wittmann, K. Kremer, and K. Binder, J. Chem. Phys. 96, 6291 (1992).

[4] A. Coniglio, H. E. Stanley, and W. Klein, Phys. Rev. B 25, 6805 (1982).

[5] H. E. Stanley, R. L. Blumberg, and A. Geiger, Phys. Rev. B 28, 1626 (1983).

[6] F. Sciortino, P. H. Poole, H. E. Stanley, and S. Havlin, Phys. Rev. Lett. 64, 1686 (1990).

[7] C. Delisi and A. S. Perelson, J. Theor. Biol. 62, 159 (1976).

[8] R. J. de Boer, L. A. Segel, and A. S. Perelson, J. Theor. Biol. 155, 295 (1992).

[9] J. Stewart and F. J. Varela, J. Theor. Biol. 153, 477 (1991).

[10] S. Torquato, Random Heterogeneous Materials (SpringerVerlag, New York, 2001).

[11] Statistical Models for the Fracture of Disordered Media, edited by H. J. Herrmann and S. Roux (North-Holland, Amsterdam, 1990).

[12] M. Sahimi, Rev. Mod. Phys. 65, 1393 (1993).

[13] P. J. E. Peebles, The Large Scale Structure of the Universe (Princeton University Press, Princeton, NJ, 1980).

[14] P. E. Seiden and L. S. Schulman, Adv. Phys. 39, 1 (1990).

[15] W. C. Saslaw, The Distribution of Galaxies (Cambridge University Press, Cambridge, England, 2000).

[16] M. Sahimi, Applications of Percolation Theory (Taylor and Francis, London, 1994)

[17] G. Grimmett, Percolation (Springer-Verlag, Berlin, 1999).

[18] T. L. Hill, J. Chem. Phys. 23, 617 (1955).

[19] A. Coniglio, U. De Angelis, A. Forlani, and G. Lauro, J. Phys. A 10, 219 (1977).

[20] L. A. Pugnaloni and F. Vericat, J. Chem. Phys. 116, 1097 (2002).

[21] For example, in the conductor-insulator transition of water in oil microemulsions, two micelles are consider bonded if they can share charge carriers (see Ref. [22]). However, in the iden- tification of bridges formed by grains within granular materials, two grains are bonded if they stabilize each other (see Ref. [23]).

[22] S. H. Chen, J. Rouch, F. Sciortino, and P. Tartaglia, J. Phys.: Condens. Matter 6, 10855 (1994).

[23] L. A. Pugnaloni, G. C. Barker, and A. Mehta, Adv. Complex Syst. 4, 289 (2001).

[24] L. A. Pugnaloni, I. F. Marquez, and F. Vericat, Physica A 321, 398 (2003).

[25] Y. C. Chiew and E. D. Glandt, J. Phys. A 16, 2599 (1983).

[26] T. De Simone, S. Demoulini, and R. M. Stratt, J. Chem. Phys. 85, 391 (1986).

[27] D. Laría and F. Vericat, Phys. Rev. A 43, 1932 (1991).

[28] F. H. Stillinger, J. Chem. Phys. 38, 1486 (1963).

[29] F. W. Starr, J. K. Nielsen, and H. E. Stanley, Phys. Rev. Lett. 82, 2294 (1999).

[30] L. A. Pugnaloni, cond-mat/0406713.

[31] L. A. Pugnaloni and F. Vericat, Phys. Rev. E 61, R6067 (2000).

[32] J. P. Hansen and I. R. McDonald, Theory of Simple Liquids (Academic Press, London, 1976).

[33] F. Lado, Phys. Rev. E 55, 426 (1997).

[34] A. Ben Naim, Water and Aqueous Solutions (Plenum Press, New York, 1974).

[35] J. M. J. van Leeuwen, J. Groeneveld, and J. De Boer, Physica (Amsterdam) (Amsterdam) 25, 792 (1959).

[36] C. G. Gray and K. E. Gubbins, Theory of Molecular Fluids (Clarendon, Oxford, 1984).

[37] P. M. Morse and H. Feshbach, Methods of Theoretical Physics (McGraw-Hill, New York, 1953).

[38] Handbook of Mathematical Functions, edited by M. Abtramowitz and I. A. Stegun (Dover, New York, 1965), Chap. 22.

[39] A. Z. Panagiotopoulos, Mol. Phys. 61, 813 (1987).

[40] G. Stell, J. Phys.: Condens. Matter 8, A1 (1996).

[41] D. M. Heyes and J. R. Melrose, Mol. Phys. 66, 1057 (1989).

[42] C-Y. M. Tung and P. J. Dynes, J. Appl. Polym. Sci. 27, 569 (1982). 